\title{
Layer-by-layer assembly of epidermal growth factors on polyurethane films for wound closure
}

\author{
Abhilash Kulkarni', William Diehl-Jones ${ }^{2}$, Sadegh Ghanbar ${ }^{3}$ and Song Liu ${ }^{1,3}$
}

\begin{abstract}
To facilitate the healing of chronic wounds, growth factors such as epidermal growth factor need to be safely encapsulated for their sustained and effective delivery to the wound bed. Using a layer-by-layer assembly technique, epidermal growth factor is successfully encapsulated on the surface of poly(acrylic acid)-modified polyurethane film. The amount of encapsulated epidermal growth factor is controlled by adjusting the number of chitosan/epidermal growth factor bilayers. A controlled release of epidermal growth factor from the surface of polyurethane film for a period of five days is achieved with well-retained bioactivity (over $90 \%$ ) as evidenced by a cell proliferation assay. In an in vitro cellular wounding assay, the cell gap covered with the epidermal growth factor-loaded polyurethane film closes at a rate more than twice as fast as that covered with a control polyurethane film. Fluorescent staining of F-actin reveals that the released epidermal growth factor induces differences in cytoskeletal organization, suggesting that stimulated cell migration also contributes to the close of the cell gap.
\end{abstract}

Keywords

Delivery of EGF, PU film, LbL assembly, wound healing

\section{Introduction}

Chronic wounds are a worldwide health problem and represent a significant social and financial burden to the healthcare system. In the United States alone, approximately $15 \%$ of diabetes patients develop skin ulcers, ${ }^{1}$ and many of the more than 60,000 amputations per year are sequel of poor wound healing. ${ }^{2}$ The costs associated with the care of skin disease or wound care are very significant. Medical costs associated with wound care are as much as $\$ 36,000$ per patient. Overall costs associated with chronic wounds, including loss of productivity, are estimated to be $\$ 20$ billion per year. ${ }^{3}$

Wounds which require couple of months or longer to resolve or which display dysfunctional healing are described as chronic wounds. ${ }^{4,5}$ Typically, such wounds do not generate functional granulation tissue and remain in an inflammatory stage. For example, even with "good" wound care, over 20 weeks only $31 \%$ of diabetic foot ulcers healed fully. ${ }^{6}$ To address these issues, the use of recombinant growth hormones in chronic wounds is currently being explored, which offers interesting possibilities for modulating the wound microenvironment. Specifically, epidermal growth factor (EGF) has evinced high-affinity receptors in both cells-keratinocytes and fibroblasts. EGF can also stimulate the production of collagen and accelerate wound healing. ${ }^{7-11}$

Several clinical studies have demonstrated that an important and often underappreciated aspect of the wound healing paradigm is the effective delivery of polypeptides to the wound site. These polypeptides suffer degradation during delivery, especially in chronic wounds where active inflammation results in the expression of large quantities of proteases. For example, the application of both acidic fibroblast growth factor (aFGF) and basic fibroblast growth factor (bFGF) in

\footnotetext{
'Department of Textile Sciences, Faculty of Human Ecology, University of Manitoba, Winnipeg, Manitoba, Canada

${ }^{2}$ Faculty of Health Disciplines, Athabasca University, Athabasca, Alberta, Canada

${ }^{3}$ Department of Chemistry, Faculty of Science, University of Manitoba, Winnipeg, Manitoba, Canada
}

\section{Corresponding author:}

Song Liu, Department of Textile Sciences, Faculty of Human Ecology, University of Manitoba, Winnipeg, Manitoba R3T 2N2, Canada.

Email: Song.Liu@umanitoba.ca 
the therapy of the healing-impaired wound has not always been successful, due to their high diffusibility and short half-life in vivo. ${ }^{12,13}$ EGF, another key growth factor required for wound healing, also has a relatively short half-life of approximately $1 \mathrm{~h}$ in vivo and is physically unstable. ${ }^{14-16}$ Further complicating this issue, in vivo animal studies have demonstrated the need for long-term exposure to EGF to achieve a complete cellular response. ${ }^{17}$ The best means to facilitate this appears to be by a controlled release system, which can also obviate the need for frequent dressing changes. The later can heighten the risk of nosocomial infection $^{18}$ and increase patient pain, cost, and the potential for delayed wound reepithelialization.

In short, if growth factors are to be delivered to chronic wounds, some form of encapsulation is required. We report herein an optimized delivery system that utilizes the layer-by-layer (LbL) assembly technique which is particularly effective method for fabricating tunable nanoscale multilayers on functionalized surfaces and which allows for the controlled release of growth factors.

Growth factors have been encapsulated in various biomedical materials such as nanofibers ${ }^{19,20}$ and microspheres ${ }^{21-23}$ for their sustained delivery. However, these technologies involve organic solvent, high electric voltage, or high mechanical stress which may inactivate EGF during the preparation process. LbL is a mild and effective technique for the fabrication of nanoscale multilayers on functionalized surfaces. The basic principle of this technique is the alternate deposition of polyelectrolytes with opposite charges on a charged surface via electrostatic interactions. This technique has previously been used to immobilize fibroblast growth factors (FGFs) on glass substrates, tissuecultured polystyrene, and titanium. ${ }^{24,25}$ The encapsulation of a small growth factor, EGF using the LbL technique, however, has not been reported even though an attempt has recently been made to electrostatically incorporate plasmid human EGF into linear poly(ethyleneimine) immobilized nanofibers. ${ }^{26}$

We intended to encapsulate EGF on a functionalized polyurethane (PU) film using the LbL method, to study the release of the encapsulated EGF and to evaluate its effect in the rate of cell gap closing in an in vitro cellular wound model consisting of monolayer fibroblast cells. Our hypothesis was that the mild encapsulation conditions would preserve the bioactivity of EGF and that encapsulated EGF could be released in a controlled manner to facilitate closure of fibroblast monolayer wounds. We demonstrate herein that the LbL encapsulation technique permits an effective encapsulation and controlled release of EGF with well-retained bioactivity; thus, lower concentrations of EGF were sufficient to significantly enhance the closure rates of fibroblast monolayer wounds. Also, the EGF stock solution used for the LbL assembly can be used repeatedly, minimizing the waste or loss of this expensive protein. Thus, this novel use of the LbL technique for the encapsulation of EGF with highly preserved bioactivity constitutes a promising, cost-effective tool for enhancing wound healing.

\section{Experimental section}

\section{Reagents and materials}

PU film ( $1 \mathrm{mil}=0.0254 \mathrm{~mm}$ thick $)$ was obtained from American polyfilms (Branford, CT) as a gift. A sample of a recombinant human EGF was kindly donated by Zyme Fast Inc. (Winnipeg, MB). Acrylic acid (AA), benzophenone (BP), $\quad N, N^{\prime}$-methylenebisacrylamide (MBA), and chitosan $(\mathrm{CH})\left(M_{\mathrm{W}}=70,000\right)$ were purchased from Sigma-Aldrich (Oakville, ON). Human dermal fibroblast cells (ATCC, PCS 201-010) and Quantikine ${ }^{\circledR}$ human EGF ELISA kit were purchased from Cedarlane (Burlington, ON), and MTS (3-(4,5dimethylthiazol-2-yl)-5-(3-carboxymethoxyphenyl)-2(4-sulfophenyl)-2 H-tetrazolium) cell proliferation kit was purchased from Promega (Madison, WI).

\section{Surface modifications of films and silicon wafers}

Immobilization of carboxylic acid groups on PU films. Anionic (carboxyl) groups were immobilized onto PU films to act as anchorage points for the immobilization of EGF in PU films. These films were surface-modified with AA to form an interpenetrating network. ${ }^{27}$ Briefly, a PU film was first swollen in methanol solution of AA, MBA (a cross linking agent), and BP (a photo initiator) for $1 \mathrm{~h}$ at $40^{\circ} \mathrm{C}$. The swollen film was then exposed to UV irradiation $(365 \mathrm{~nm})$ for $2 \mathrm{~h}$ to effect the formation of interpenetrating polymer network (IPN), and finally the unreacted monomer and loosely attached polymer were removed by washing the film in ethanol solution for $8 \mathrm{~h}$. The resultant PU film was referred to as "PAA-PU."

Immobilization of carboxylic acid groups onto silicon wafers. Silicon wafers were used as a model substrate for the study of $\mathrm{LbL}$ deposition of $\mathrm{CH} / \mathrm{EGF}$ polyelectrolytes. A $4 \times 4 \mathrm{~cm}^{2}$ silicon wafer was coated with PAA by plasma polymerization in a USB 8 Plasma Activation \& PECVD coating system (Plasma Technology, Reading, PA). Processing conditions were as follows: frequency, $13.56 \mathrm{MHz}$; chamber pressure, $0.3 \mathrm{mbar}$; and processing duration, $9 \mathrm{~min}$.

LbL of polyelectrolytes. To achieve LbL encapsulation of $\mathrm{EGF}$, a cationic polyelectrolyte $\mathrm{CH}$ was chosen to form 
an ion pair with EGF. CH and EGF dipping solutions were prepared using ultrapure water with a resistivity of $18.2 \mathrm{M} \Omega \cdot \mathrm{cm}$ at $25^{\circ} \mathrm{C}$ from a Milli-Q plus (Bedford, MA). $\mathrm{CH}$ was first dissolved in 3\% acetic acid and then the solution was neutralized with $1 \mathrm{M} \mathrm{NaOH}$ to get a final $\mathrm{pH}$ of 5.8 and the final $\mathrm{CH}$ concentration of $0.8 \mathrm{mg} / \mathrm{ml}$. EGF was dissolved in $\mathrm{pH} 7.4$ phosphate-buffered saline (PBS) solution at the concentration of $100 \mu \mathrm{g} / \mathrm{mL}$.

Both silicon wafers and modified PU films were cut to the dimension of approximately $2 \times 2 \mathrm{~cm}^{2}$ before $\mathrm{LbL}$ assembly of $\mathrm{CH}$ and EGF. The substrates were first dipped into $\mathrm{CH}$ solution and allowed to sit for $15 \mathrm{~min}$. Afterwards, the substrates were rinsed three times in ultrapure water. Then the charge on the substrate was reversed by dipping them into EGF solution for $15 \mathrm{~min}$ and rinsing for three times using ultrapure water. This procedure was repeated for a predetermined number of times depending on the experimental design. Once the required number of layers was deposited onto the substrates, they were dried at $30^{\circ} \mathrm{C}$ under vacuum for $30 \mathrm{~min}$.

\section{Characterization of the modified film and wafer}

A colorimetric titration was used to quantify the carboxyl density on PAA-PU. The growth of $\mathrm{CH} / \mathrm{EGF}$ was monitored using contact angle measurements at each layer, atomic force microscope (AFM), and ellipsometry at every five layers. Before subjecting these wafers for measurements, the wafers were dried and all superficial dirt was removed using a nitrogen gun.

Colorimetric titration. In order to quantify the amount of carboxyl group on PAA-PU substrates, a colorimetric titration technique was employed. PU films were immersed in $5 \mathrm{~mL}$ of ethanol solution of thionine acetate dye $(0.1 \mathrm{mg} / \mathrm{mL})$ and then shaken for overnight at room temperature. ${ }^{28}$ The films were then removed and washed with ethanol for three times until nonspecifically adhered dye molecules were removed. The absorbance of residual dye solutions and rinsing solutions were measured and compared with that of the initial dye solution. The difference obtained was used for the determination of the concentration of carboxyl group on PAA-PU substrates.

Contact angle measurement. Contact angle measurement was used to monitor the change in the hydrophilicity of the modified wafer surface. After one layer of polyelectrolyte was deposited, the wafer sample was rinsed with ultrapure water, dried at $30^{\circ} \mathrm{C}$ for $10-15 \mathrm{~min}$, and then small size dust particles were removed using a nitrogen gun. Contact angle measurement was then made using a Rame-hart Model 200 goniometer $(n=5)$. A $2-\mu \mathrm{L}$ droplet was applied from a syringe onto the sample and the contact angle was determined.
Atomic force microscope. Silicon wafer samples were investigated using a Nanoscope III scanning probe microscope (Veeco, D3100) in tapping mode after purging the samples with a nitrogen gun. Nanoscope's commercially available $\mathrm{n}+$ silicon cantilevers with a force constant of $10-130 \mathrm{~N} / \mathrm{m}$ and a resonance frequency around $300 \mathrm{KHz}$ were used for measuring the surface topography. The images were scanned through an area of $10 \times 10 \mu \mathrm{m}$ and then zoomed into $3 \times 3 \mu \mathrm{m}$.

Ellipsometry. In order to determine the thickness of the deposited polyelectrolytes, ellipsometry measurements were performed using J.A. Woollam (Model M2000D) at a wavelength of $500 \mathrm{~nm}$ and at incident angles of $55^{\circ}, 65^{\circ}$, and $75^{\circ}$. The polyelectrolyte layers were built on a silicon wafer. Before depositing the layers on the wafer, the wafer was cleaned using soap solution and continuously rubbed with a scrubber for 5 min. Twenty layers $(\mathrm{CH} / \mathrm{EGF})_{10}$ were built on the silicon wafer as mentioned above. The thickness was determined by using a two-layer model. The first layer is silicon oxide whose thickness is a constant, and the second layer is the deposited polyelectrolyte layers measured at an interval of five layers till 20 layers of deposition. On each sample, 20 different positions were chosen, and the thickness measurement was performed and calculated as the average of all these measurements.

\section{Release of EGF}

$\mathrm{CH} / \mathrm{EGF}$-deposited PAA-PU film was used for the release study. Ten layers $(\mathrm{CH} / \mathrm{EGF})_{5}$ and 40 layers $(\mathrm{CH} / \mathrm{EGF})_{20}$ were built on PAA-PU film and the films were stored at $-20^{\circ} \mathrm{C}$ until use. The films with a size of $2 \times 2 \mathrm{~cm}^{2}$ were immersed into $10 \mathrm{~mL}$ of PBS solution in a tightly sealed vial to prevent any evaporative loss of water and EGF was released in this media in a thermostat water bath $\left(37^{\circ} \mathrm{C}\right)$. Two milliliters of the solution was removed from the above solution at predetermined time points and this $2 \mathrm{~mL}$ was replaced with $2 \mathrm{~mL}$ of fresh PBS solution. The cumulative release of EGF was monitored for a period of seven days.

The concentration of the released EGF was determined by enzyme linked immunosorbent assay (ELISA) using a Human EGF Quantikine ELISA kit (R\&D systems, Minneapolis, MN, USA). ${ }^{29,30}$ All the measurements were conducted in triplicate.

\section{Bioactivity of released EGF}

Cell proliferation test was used to evaluate the bioactivity of the released EGF in respect to the native EGF. ${ }^{31,32}$ Human neonatal fibroblast cells were cultured in fibroblast basal serum-free media and $1 \%$ penicillin-streptomycin in a $37^{\circ} \mathrm{C}, 5 \% \mathrm{CO}_{2}$ environment. 
$2 \times 10^{5}$ cells were seeded onto a 96-well plate in serumfree media before released EGF samples were added. MTS assay kit (CellTiter 96 Nonradioactive Cell proliferation assay, Promega, Madison, WI) was used to evaluate the bioactivity of released EGF. Once the cells were attached and confluent, the released EGF and native EGF which was prepared at the same concentration of the released EGF were added into each well and incubated for $24 \mathrm{~h}$. MTS/PMS complex $(20 \mu \mathrm{L})$ was then added into these wells, and cell proliferation was measured after $4 \mathrm{~h}$ at $490 \mathrm{~nm}$ using an Opsys microplate reader. We chose this method for testing the bioactivity of released EGF, as opposed to culturing cells directly on film, due to the targeted wound dressing application where EGF should be released into the wound. The released EGF was diluted 10 times in cell medium before being added into the wells in order to minimize possible interferences of PBS solution into which EGF was released. Percent of retained bioactivity of EGF was calculated as $A / A_{0}$, where $A$ and $A_{0}$ were the absorbencies $(490 \mathrm{~nm})$ of cells cultured in the released and native EGF solutions, respectively (note: both solutions had the same concentration of EGF).

\section{Actin staining}

Cells were grown to $80 \%$ confluence on collagen-coated glass cover slips. Three different treatments were used: (i) no serum, (ii) regular medium (fibroblast basal medium), and (iii) medium with released EGF. Cells were starved without any serum in all cases for one day prior to experiments. Cells in the third treatment group were incubated with released EGF at a concentration of around $800 \mathrm{pg} / \mathrm{mL}$ and incubated for $3 \mathrm{~h}$. Cells were next washed five times with PBS at $\mathrm{pH} 7.2$ and fixed for 10 min using 4\% paraformaldehyde (v/v) in PBS. Samples were washed three times with PBS and permeabilized in ice cold acetone for $3-5 \mathrm{~min}$. Finally the cells were labeled with Alexa Fluor 488 Phalloidin (Invitrogen), a stain which is specific for filamentous (F)-actin in a darkened place for $30 \mathrm{~min} .{ }^{33,34}$ Cover slips were mounted on cleansed glass slides and viewed with a fluorescence microscope (Zeiss Photo II) using neoflor objectives using $20 \times$ and $40 \times$ objective lens. Images were captured with a Retiga Q-imaging camera and stored using Northern Eclipse software (Empix Imaging, Mississauga, ON). Gain, offset, and exposure times were identical for all treatment groups.

\section{Fibroblast monolayer wound model}

A simple fibroblast monolayer wound model consisting of a layer of human neonatal fibroblast cells was cultured with a seeding density of $3 \times 10^{5}$ in $35-\mathrm{mm}$ round Petri dishes coated with Type-I collagen and incubated at $37^{\circ} \mathrm{C}, 5 \% \mathrm{CO}_{2}$ till $90-95 \%$ confluence was reached, and then an incision was made in the cell layer using a pipette tip. EGF-incorporated $(\mathrm{CH} / \mathrm{EGF})_{20} \mathrm{PU}$ film and control film were laid above the cells containing media and cell monolayer wound closure was measured under the microscope over a time period till the wound was completely closed. For each dish, two to three scratches were made using a $10-100 \mu \mathrm{L}$ pipette tip, resulting in well-defined scratch which did not induce any noticeable deformations in the polycarbonate substrate. Detached cells were then washed away using a cell culture medium. Films were prewetted in the cell media for $2 \mathrm{~min}$ in $2 \mathrm{~mL}$ of cell media and then the media with film were added into these dishes with the film completely covering the cell layer. Cells growing on Petri dishes were maintained in an enclosed heating stage at $37^{\circ} \mathrm{C}$ and with $5 \% \mathrm{CO}_{2}$. The well-defined scratch was photographed every $5 \mathrm{~min}$ using a Zeiss Axiovert 200 inverted microscope with a $10 \times$ planapo objective until the wound was closed. Phase contrast images were captured using a $16 \times$ planapo objective len and a Retiga Q-imaging camera. Optical calipers were used to measure wound closure rates using Northern Eclipse software.

\section{Result and discussion}

\section{LbL assembly of CH/EGF bilayer on PAA-modified PU film}

PU film was chosen as a representative substrate due to its biocompatibility, hemocompatibility, and flexibility. To enable LbL assembly of EGF on PU film, positive or negative charge should be first introduced into the surface. AA was copolymerized with a crosslinker MBA in the methanol swollen surface of PU film to form an IPN for the durable immobilization of PAA according to the articles previously published by our research group. ${ }^{27,35}$ Fourier transform infrared spectra of treated and untreated PU films are presented in Figure 1. Unlike in the spectrum of untreated PU film (Figure 1a) where two peaks $\left(1724\right.$ and $1700 \mathrm{~cm}^{-1}$ ) had similar intensities, the $1700 \mathrm{~cm}^{-1}$ peak in the spectrum of PAA-modified PU film (PAA-PU) seemed to be spiked higher than $1724 \mathrm{~cm}^{-1}$ which is characteristic of carbamate bond in PU film (Figure 1b). After subtraction, the peak at $1695 \mathrm{~cm}^{-1}$ stood out indicating the presence of carboxylic acid group. The surface density of carboxylic acid group was further quantified to be $330 \mathrm{nmol} / \mathrm{cm}^{2}$ using a colorimetric titration method.

The stability of EGF had been studied as a function of buffer $\mathrm{pH}$ and temperature. ${ }^{36,37}$ Higher temperature leads to faster degradation of EGF. The $\mathrm{pH}$ of its maxim stability shifts from 5.6 at higher temperature $\left(50-60^{\circ} \mathrm{C}\right)$ to 7.2 at lower temperature $\left(25-40^{\circ} \mathrm{C}\right) .^{37}$ 


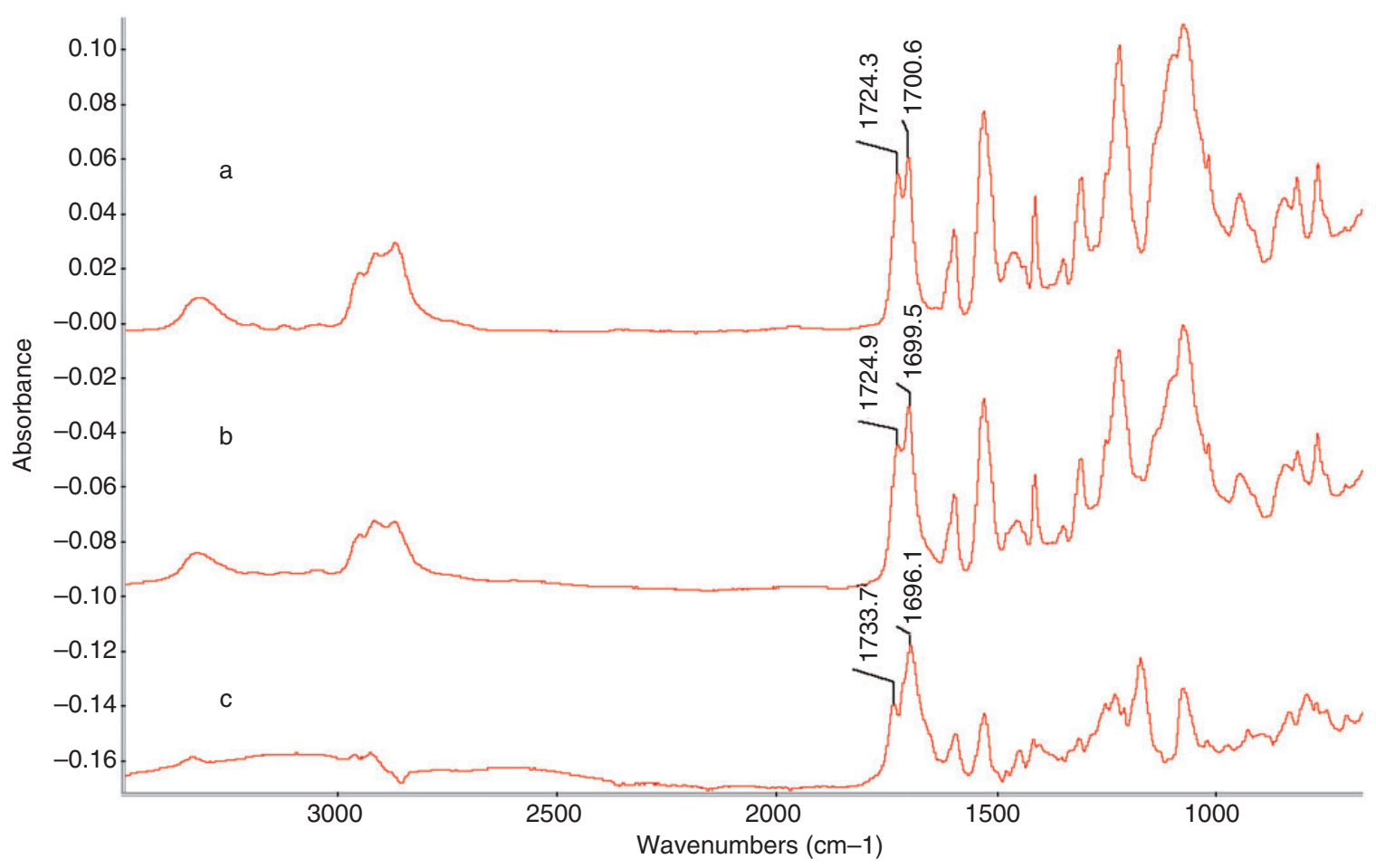

Figure I. Fourier transform infrared spectra of (a) untreated PU film, (b) PAA-modified PU film, and (c) subtraction of (a) from (b).

So, it is desirable to conduct the encapsulation EGF at room temperature at the biological $\mathrm{pH}$ (7.4) for the maximum retention of its bioactivity. EGF is a weak polyelectrolyte whose charge highly depends on the $\mathrm{pH}$. With an isoelectric point of $4.60,{ }^{38,39} \mathrm{EGF}$ is negatively charged at $\mathrm{pH}$ 7.4. A polycation counterpart is required for the LbL assembly. It is known that polyelectrolyte complexes formed by protein and weak polyions could gradually dissociate in solutions with a $\mathrm{pH}$ close to the isoelectric point of one of the components. To allow the later release of encapsulated EGF, a weak polyanionic polyelectrolyte should be adopted. $\mathrm{CH}$, a biodegradable polymer derived by deacetylation of the naturally occurring polysaccharide chitin, is a weak polyelectrolyte. Importantly, $\mathrm{CH}$ has been proven to possess antimicrobial activity which is also beneficial for wound healing. Therefore, $\mathrm{CH}$ was chosen as the polycation counterpart of EGF in the LbL assembly.

Charge density is a critical parameter for alternate adsorption of oppositely charged polyelectrolytes. $\mathrm{CH}$ has an isoelectric point at $\mathrm{pH} 6.3^{40,41}$ with a high charge density at $\mathrm{pH} 4$, but the bioactivity of EGF might be compromised at that $\mathrm{pH}$. To better preserve the bioactivity of EGF, $\mathrm{pH}$ of $\mathrm{CH}$ deposition solution was raised to 5.8 at which the charge density of $\mathrm{CH}$ drops to $50-60 \%(50-60 \%$ of $\mathrm{CH}$ repeating units are still positively charged in the solution). ${ }^{42,43}$ This was chosen since as little as $20 \%$ of charged blocks per chain could enable multilayer formation. ${ }^{44}$
Contact angle test was adopted to monitor the growth of $\mathrm{CH} / \mathrm{EGF}$ bilayers on PU film, i.e., the encapsulation of EGF. Contact angle measurements was conducted using a Rame-hart Model 200 goniometer $(n=5)$. As shown in Figure 2, PAA-PU film became more hydrophilic with a lower contact angle $(37.5 \pm 2.4)$ than the untreated PU film $(97.1 \pm 1.7)$. Increase of contact angle to $73^{\circ}$ after the first dip coating in $\mathrm{CH}$ solution indicated a successful deposition of $\mathrm{CH}$ layer on the surface of PAA-PU film. The uniform coverage of $\mathrm{CH}$ was evidenced by the small variation of the measurement (Figure 2). A significant decrease of contact angle after the subsequent EGF assembly suggested the formation of a more hydrophilic EGF layer on the top. Alternate $\mathrm{CH} / \mathrm{EGF}$ assembly was achieved up to 10 layers as supported by the reciprocal change of contact angles.

\section{LbL assembly of CH/EGF bilayer on PAA coated sili- con wafer}

In order to understand the topography of the $\mathrm{CH} / \mathrm{EGF}$ bilayers, and to estimate the thickness formed by these bilayers, a model substrate silicon wafer was used instead of PU film which cannot be easily prepared for AFM measurement due to its elasticity and flexibility (only 1 mil thick). Silicon wafer was coated with AA by plasma polymerization. AA was polymerized onto silicon wafer under the influence of plasma. 


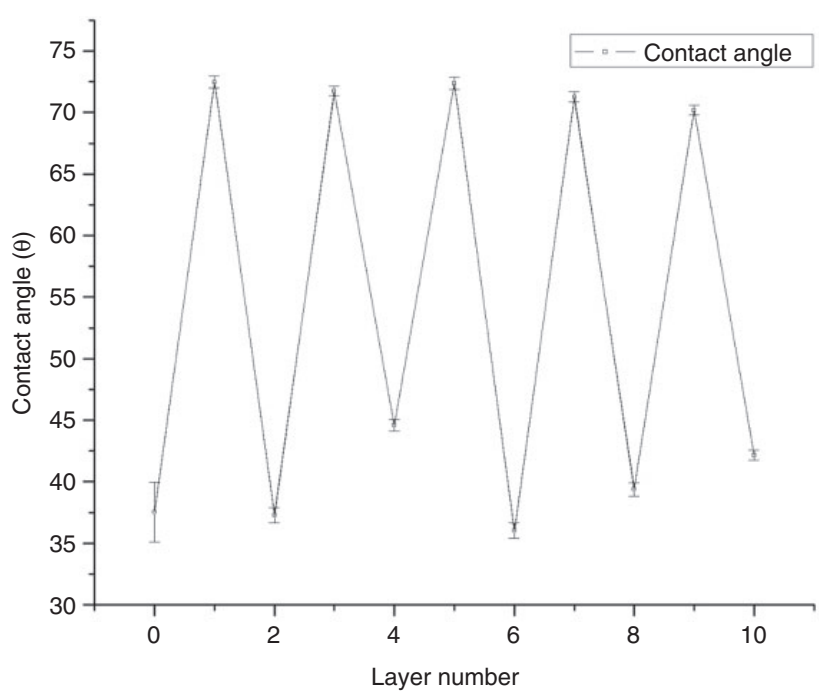

Figure 2. Contact angle as a function of $\mathrm{CH} / \mathrm{EGF}$ assembled on PAA-PU film ( 10 layers). Odd numbers indicate the measurements for $\mathrm{CH}$ and even numbers represent EGF measurements.

Subsequently, $\mathrm{CH} / \mathrm{EGF}$ bilayers were deposited following the same protocol. The results of contact angle measurement (Figure S1, Supporting Information) lent support to the formation of the polyelectrolyte bilayer up to 15 layers.

AFM was employed to verify the deposition of the polyelectrolytes at regular intervals. The multilayers were scanned in tapping mode after the surfaces were gently blown with a stream of nitrogen. As can be seen from Figure 3, the surface roughness did not change significantly upon the deposition of PAA while the surface did become rougher upon the deposition of $\mathrm{CH} /$ EGF. The root mean square (RMS) of the oscillation amplitude of AFM probe can be used as a quantitative measurement of surface roughness and is presented in Table 1. The RMS increased with the growth of polyelectrolyte film, from $0.355 \mathrm{~nm}$ (PAA coated silicon wafer) to $2.20 \mathrm{~nm}$ for five polyelectrolyte layers, to $2.80 \mathrm{~nm}$ for 10 layers, and to $3.02 \mathrm{~nm}$ for 15 layers. The raise in the roughness after the deposition of five polyelectrolyte layers indicated the deposition of $\mathrm{CH}$ and EGF molecules. With further deposition, the slope of RMS increase became less steep. One plausible reason is that further deposited polyelectrolytes cover the gaps formed in preceding layers. Clearly, globular conformations or peaks appeared as the LbL film was further constructed. Since "roughness" obtained from AFM does not well correlate with the thickness of the polyelectrolyte film, ellipsometry was adopted to characterize the LbL film.

As shown in Figure 4, the thickness of the CH/EGF film increased linearly with the number of layers with approximately $1.76 \mathrm{~nm}$ per bilayer, as there is no exponential growth. For big proteins such as lysozyme, the layer thickness of the protein can be as big as $4.5 \mathrm{~nm} .{ }^{45}$ Given that the hydrodynamic diameter of EGF is only $3.7 \mathrm{~nm},{ }^{46}$ it is not surprising to find that $\mathrm{CH} / \mathrm{EGF}$ bilayer thickness falls in the typical range of bilayer thickness of the linear polyions $(1.5-2 \mathrm{~nm}){ }^{47}$ Steady increase of the thickness of $\mathrm{CH} / \mathrm{EGF}$ bilayer suggests the lack of interlayer interpenetration of the polyelectrolyte in the deposition of 10 bilayers of $\mathrm{CH} /$ EGF. The characterization of $\mathrm{CH} / \mathrm{EGF}$ bilayer on model silica wafer gives us insights on the structure of $\mathrm{CH} / \mathrm{EGF}$ bilayers assembled in the same way on PAAPU film. To conclude, EGF could be effectively encapsulated on the $-\mathrm{COOH}$ functionalized surface with the counterion polyelectrolyte $\mathrm{CH}$ using the LbL deposition technique.

\section{In vitro EGF release from polyelectrolyte-deposited PU films}

For tissue engineering applications, the purpose of encapsulating growth factors on the surface of tissue engineering scaffold is to enhance cell attachment and proliferation. ${ }^{25}$ For the targeted wound healing application of this work, release of EGF from PU film is of interest. After the successful deposition and characterization of $\mathrm{CH} / \mathrm{EGF}$ bilayer on both PAA-PU film and model substrate silica wafer, we studied the release profile from the EGF containing PAA-PU film. First, 10 layers of polyelectrolytes, i.e., $(\mathrm{CH} / \mathrm{EGF})_{5}$ were constructed and released in vitro. Within one day, the released EGF only amounted to about $1.1 \mathrm{ng}$ as shown in Figure 5. When 40 layers of $\mathrm{CH}$ and EGF were stacked onto PAA-PU film, one day release increased significantly to $17.13 \mathrm{ng}$ and the overall release reached $22 \mathrm{ng}$. This indicated the amount of encapsulated and later released EGF can be tuned by adjusting the number of fabricated polyelectrolyte bilayers. Approximately $76 \%$ of the EGF was released in the first $24 \mathrm{~h}$, from PAA-PU film (40 layers of polyelectrolytes). This is advantageous since an initial higher dosage could help initiate cell responses in chronic wounds. The release was prolonged in a zeroorder manner for three days and reached a plateau, thereafter with $99 \%$ EGF released on day 6 . According to Almodovar et al., ${ }^{25}$ the most hydrophilic polyelectrolyte multilayers can be generated by pairing up either weak polycation with weak polyanion or strong polycation with strong polyanion. In contact with buffer solution, hydrophilic polyelectrolyte multilayers will swell to larger extents than relatively hydrophobic ones. Since both EGF and $\mathrm{CH}$ are weak polyelectrolytes, it can be expected that the $\mathrm{CH} / \mathrm{EGF}$ bilayers are very hydrophilic, facilitating EGF release. However, the relatively high percentage of EGF release 
(a) $10.0 \mathrm{~nm}$

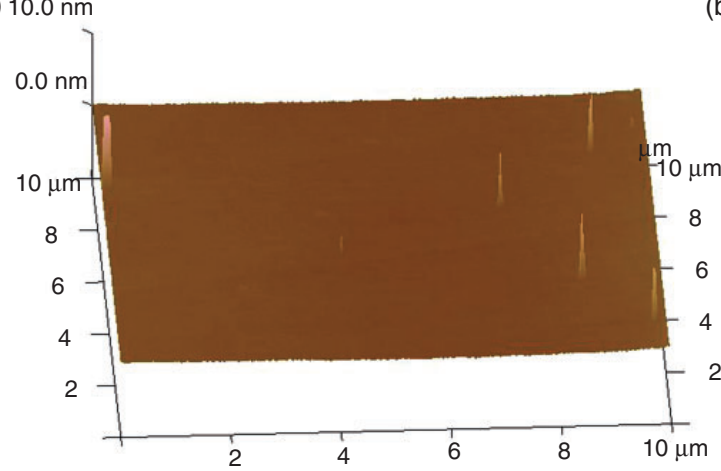

(b) $10.0 \mathrm{~nm}$

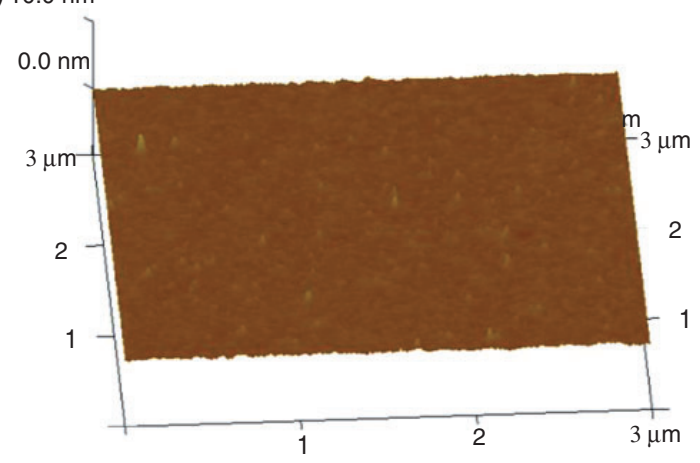

(c) $20.0 \mathrm{~nm}$

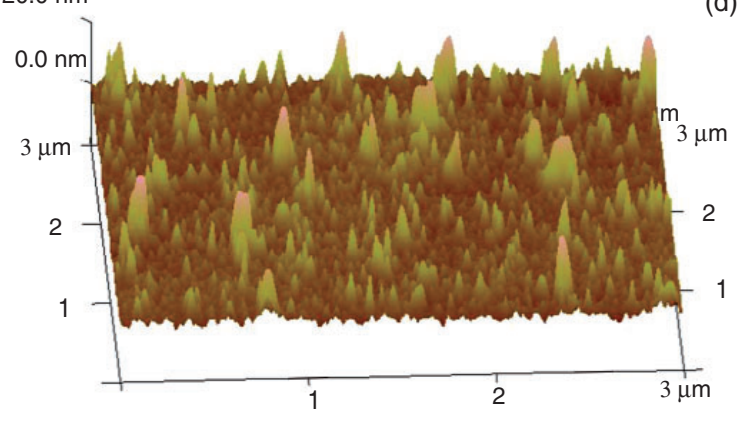

(d) $20.0 \mathrm{~nm}$

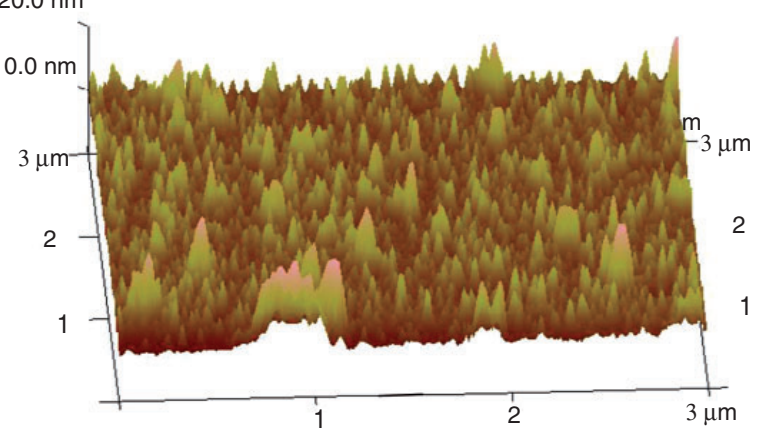

(e) $20.0 \mathrm{~nm}$

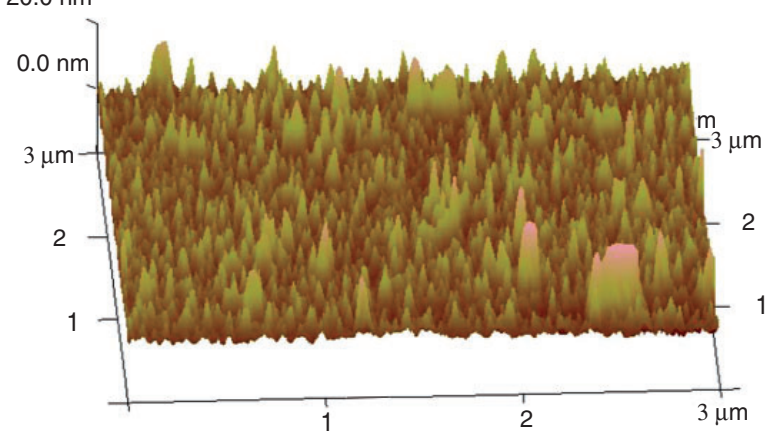

Figure 3. AFM images of (a) silicon wafer, (b) PAA-deposited silicon wafer (a layer of carboxyl group), (c) 5 layers of CH/EGF, (d) 10 layers of $\mathrm{CH} / \mathrm{EGF}$, and (e) 15 layers of $\mathrm{CH} / \mathrm{EGF}$.

Table I. Surface roughness values (RMS) of various silicon wafer samples.

\begin{tabular}{ll}
\hline Parameters & $\begin{array}{l}\text { Roughness } \\
(\mathrm{RMS})(\mathrm{nm})\end{array}$ \\
\hline Silicon wafer & 0.355 \\
Modified silicon wafer [poly(acrylic acid)] & 0.486 \\
5 Layers of CH/EGF (CH top layer) & 2.20 \\
I0 Layers of CH/EGF (EGF top layer) & 2.80 \\
I5 Layers of CH/EGF (CH top layer) & 3.02 \\
\hline
\end{tabular}

in day 1 might be majorly due to the swelling of PAAPU film. In the test of surface zeta-potential of PAAPU film using an adjustable gap cell, we observed that the gap between two pieces of PAA-PU film decreased linearly (Figure S2, Supporting Information) from around $94 \mu \mathrm{m}$ to $82 \mu \mathrm{m}$ in about $1 \mathrm{~h}$, indicating swelling of PAA-PU film. As the film swells, small electrolytes such as phosphate ions can better diffuse into the $\mathrm{CH} /$ EGF bilayers and hence dissociate the electrostatic binding between $\mathrm{CH}$ and $\mathrm{EGF}$, facilitating the release of EGF. After the swelling of PAA-PU film reached saturation, the release gradually slowed down and was sustained for several more days.

\section{Bioactivity of released EGF}

We confirmed that EGF could be encapsulated together with $\mathrm{CH}$ onto the surface of PAA-PU film using the $\mathrm{LbL}$ technique and then be sustainably released. Another critical question was whether the 
released EGF retained its biological activity. In other studies, EGF has been applied topically on wounds, and EGF has also been released in a controlled manner via nanofibers, ${ }^{19,20}$ microspheres, ${ }^{21-23}$ hydrogels, ${ }^{48}$ sol-gel, ${ }^{49}$ and pellets ${ }^{50}$ at the scale of microgram. However, in these studies, the bioactivity of the encapsulated EGF was not quantified with respect to native EGF even though similar work was done on encapsulated FGF-2. ${ }^{24}$ Our study rectifies this omission by measuring the in vitro bioactivity of the released EGF using a cell proliferation assay. The retained bioactivity was calculated according to the following equation

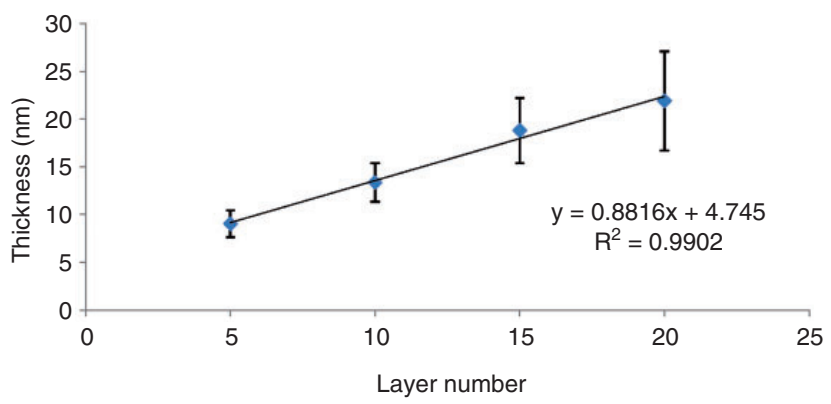

Figure 4. Thickness measurements of CH/EGF bilayers assembled on silicon wafer. (details of the test can be found in 'Bioactivity of released EGF' section):

Retained bioactivity $=$ bioactivity of released EGF as quantified by the cell proliferation assay/bioactivity of native EGF as quantified by the cell proliferation assay.

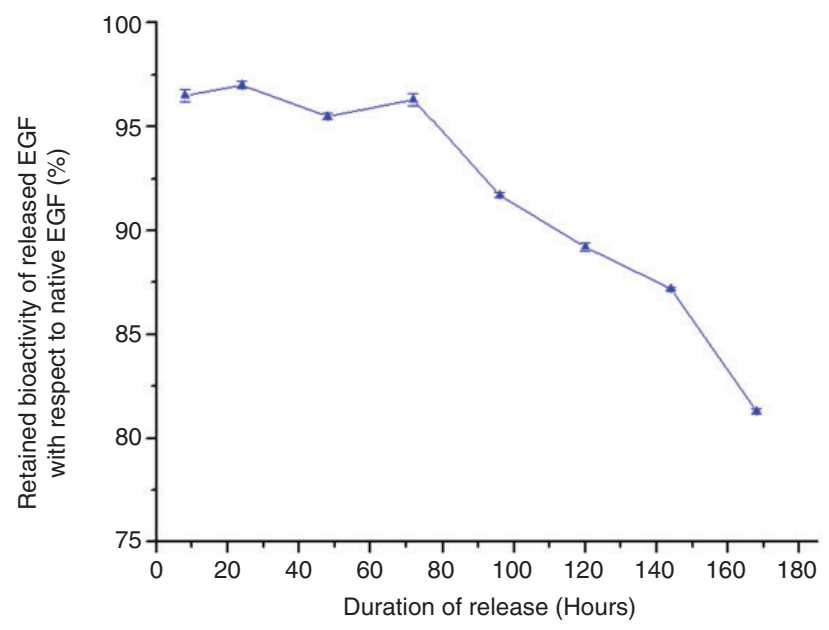

Figure 6. The percentage of retained bioactivtiy of released EGF with respect to native EGF quantified using MTS cell proliferation assay $(n=3)$.

EGF: epidermal growth factor.

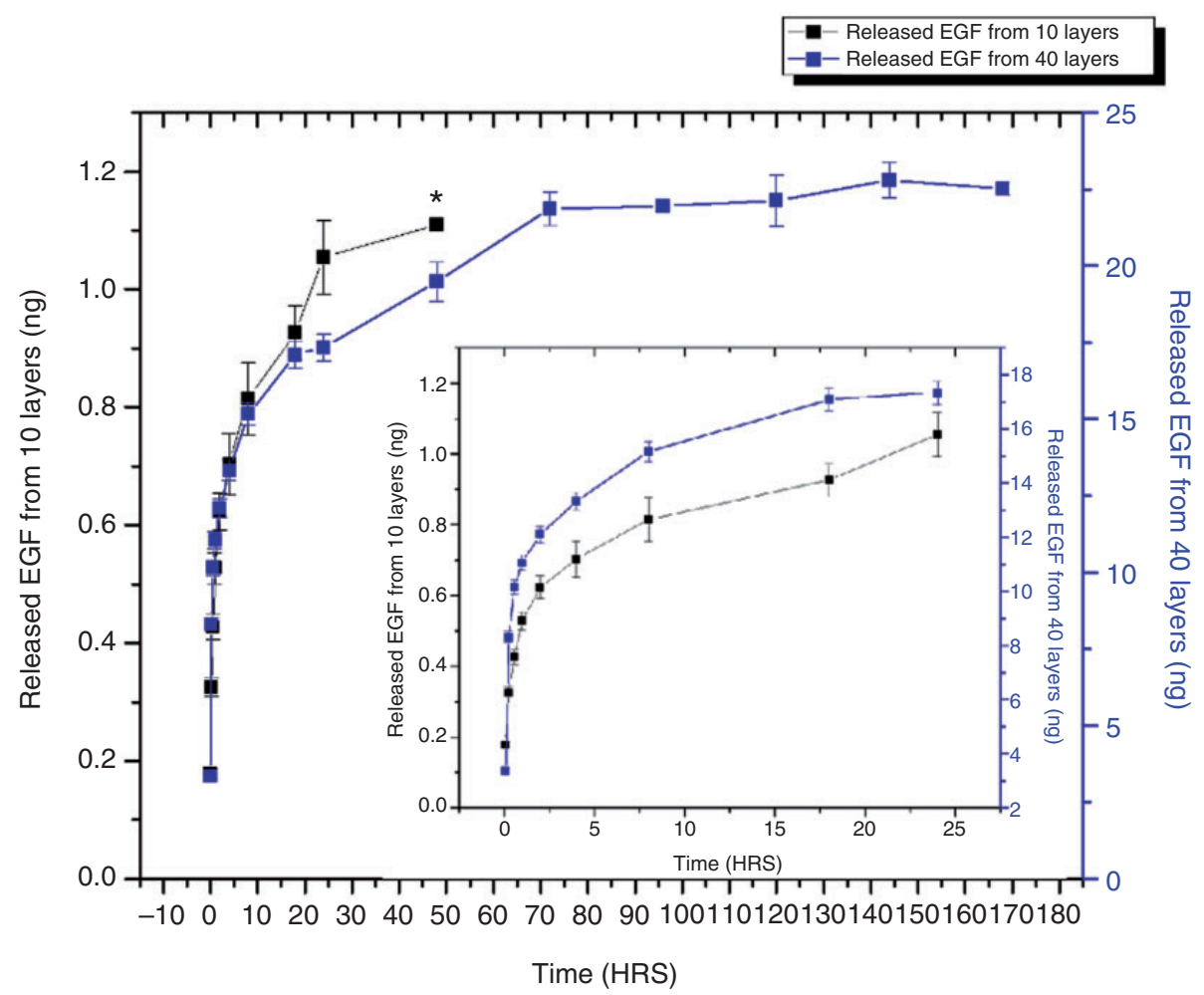

Figure 5. Release profile of EGF from 10 layers $(\mathrm{CH} / \mathrm{EGF})_{5}(\mathrm{IO} \mathrm{L})$ and 40 layers $(\mathrm{CH} / \mathrm{EGF})_{20}(40 \mathrm{~L})$. Inset shows the release profile up to $24 \mathrm{~h}$.

EGF: epidermal growth factor. 


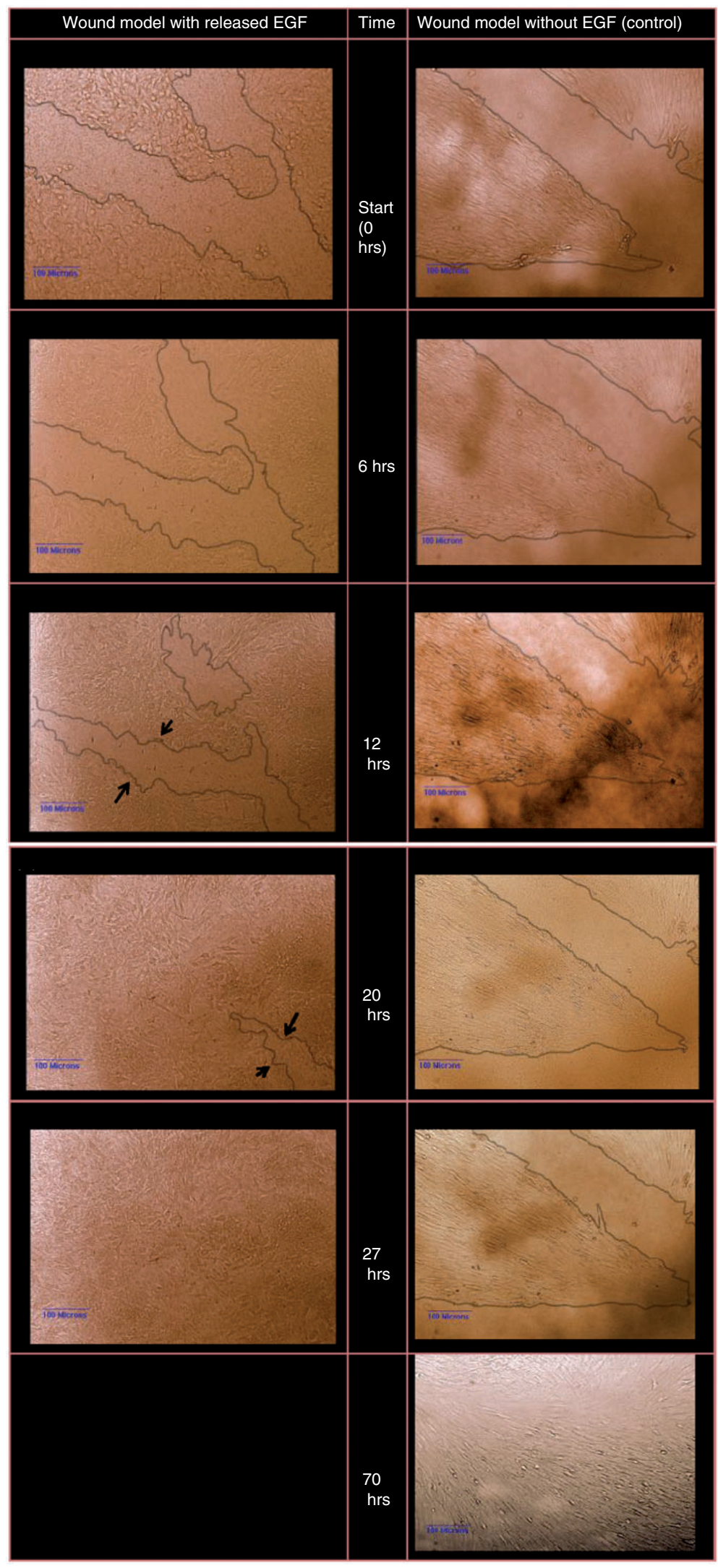

Figure 7. Healing of fibroblast monolayer model wounds covered with EGF-incorporated (CH/EGF) ${ }_{20}$ PU film and control-pristine PU film. EGF: epidermal growth factor. 


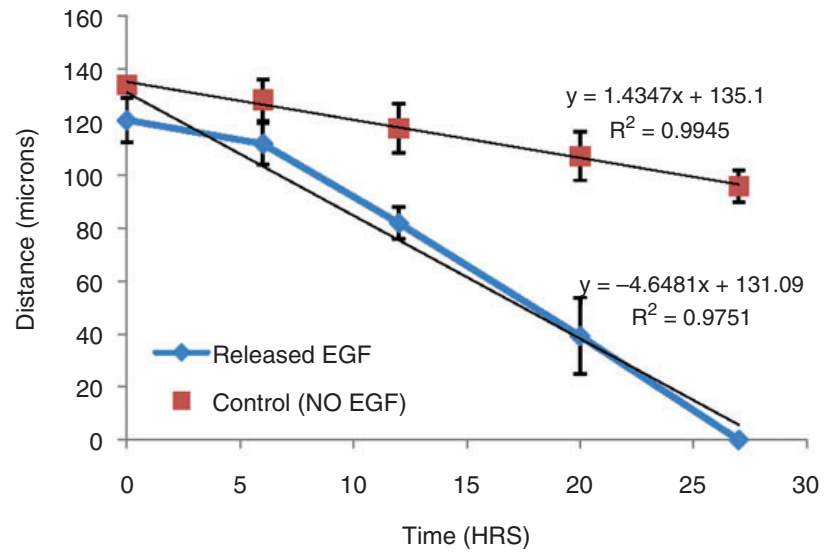

Figure 8. Fibroblast monolayer wound gap as a function of healing duration.

EGF: epidermal growth factor.

Other novel aspects of our study include the use of human neonatal fibroblasts rather than keratinocytes. Our rationale was that fibroblasts are the "first responders" in the wound healing process, and deposit the collagen substrate requisite for reepithelialization. Furthermore, nenonatal fibroblasts are generally having a higher replicative potential than adult fibroblasts, thus facilitating their ease of use and continued passaging. While not novel, the MTS assay, which was used to quantify cell proliferation, is a robust marker of the retained bioactivity of released EGF. The released EGF was compared with native EGF to obtain an evaluation of the percent of retained bioactivity by the encapsulated and released EGF.

In our system, released EGF retained a high bioactivity and induced fibroblast proliferation, albeit slightly lower than that of the native EGF. As shown in Figure 6, the released EGF was $96.9 \%$ bioactive after one day of release, and bioactivity was reduced to $90 \%$ on the fifth day and $81 \%$ on seventh day. The decreased bioactivity on released EGF is due to its intrinsic instability in PBS solution and fits well with the previously reported degradation percentage of EGF in $\mathrm{pH}$ 7.2 PBS buffer solution. ${ }^{42}$ So, the bioactivity of EGF is well preserved during the LbL encapsulation process. Given the high cost of recombinant EGF, this represents a considerable cost advantage. Even though the EGF stock solution used for the LbL assembly has a relatively high concentration $(100 \mu \mathrm{g} / \mathrm{mL})$, it can be repeatedly used to minimize the waste or loss of this expensive protein. Given that EGF in $\mathrm{pH} 7.2 \mathrm{PBS}$ is stable up to one month if stored at $-20^{\circ} \mathrm{C},{ }^{36}$ it is practical to store the EGF stock solution for its reuse on experimental days even though they are a month apart.

The other measure utilized to assess the efficiency of EGF delivery and bioactivity was closure rate of a monolayer cell "wound." A ruptured cell sample treated with EGF-incorporated $(\mathrm{CH} / \mathrm{EGF})_{20} \mathrm{PU}$ film demonstrated stimulated proliferation and migration as shown in Figure 7. The initial distance of the incision was around $120 \mu \mathrm{m}$ in case of EGF-treated samples. The images from Figure 7 indicated that the boundaries of fibroblast cells moved toward each other at a much faster rate in response to released EGF when compared to the control (PU film) where the movement was indeed slow. The cells were stimulated and migrated from one end to another as EGF was released from the film. The fast wound closure was attributed to EGF promoted migration and proliferation of the fibroblast cells. In the case of Guinea pig tracheal epithelial cells (GPTEC), addition of EGF $(2.5-15 \mathrm{ng} / \mathrm{mL}$ ) in the wound in a GPTEC monolayer created by a rubber stylet showed full closure in $12-24 \mathrm{~h},{ }^{51}$ which compares well with our results in $20 \mathrm{~h}$. In our EGF release system, the boundary of fibroblast cells migrated at a rate of $28 \mu \mathrm{m}$ per $6 \mathrm{~h}, 2.4$ times that in the case of the control $(11.7 \mu \mathrm{m}$ per 6 hours $)$ as shown in Figure 8. This 2.4-fold increase of the rate of cell gap closing can be attributed directly to the released bioactive EGF, which is well known for both its proliferative and chemotactic potential.

To rule out the possibility that $\mathrm{CH}$ could increase proliferation of fibroblast alone, we ran a $\mathrm{CH}$ dosage responsive assay (Figure S3 - Supporting Information). $\mathrm{CH}$ showed no proliferative effect on the fibroblast cells at concentration up to $1 \mu \mathrm{g} / \mathrm{mL}$, which is consistent with the previous report. ${ }^{52}$ Based on the density of $\mathrm{CH}(1.342 \mathrm{~g} / \mathrm{mL})$ and the thickness of $\mathrm{CH}$ layer on PU film $(<1 \mathrm{~nm} / \mathrm{CH}$ layer $)$, the maximum concentration of $\mathrm{CH}$, released from the $40 \mathrm{EGF} / \mathrm{CH}$ bilayers in the release medium (PBS), was estimated to be $1.07 \mu \mathrm{g} /$ $\mathrm{mL}$. Considering another 10 -fold dilution of the release EGF and $\mathrm{CH}$ mixture in cell culture medium, the final concentration of $\mathrm{CH}$ was less than $0.107 \mu \mathrm{g} / \mathrm{mL}$. Thus, the mitogenic activity of EGF encapsulated within the LbL film is preserved.

After a scratch was generated on monolayer fibroblasts, cells proliferate as well as migrate to close the gap. To take a closer look at the effect of the released EGF on the cell migration, we conducted actin staining experiments. The actin cytoskeleton is one subcellular component which is known for its role in cell migration. It should be noted that many types of F-actin subtypes are involved in the components of cell migration: formation of focal adhesions, extrusion of "lamellopodia" at the leading edge of cells, and trailing edge retractions. F-actin staining was performed to localize actin filaments using Alexa Fluor - 488 phalloidin dye, which is highly specific for F-actin. In Figure 9(a), the control samples maintained without serum exhibited a marked reduction in F-actin compared to either released EGFtreated or serum-treated cells. Cells exposed to released 

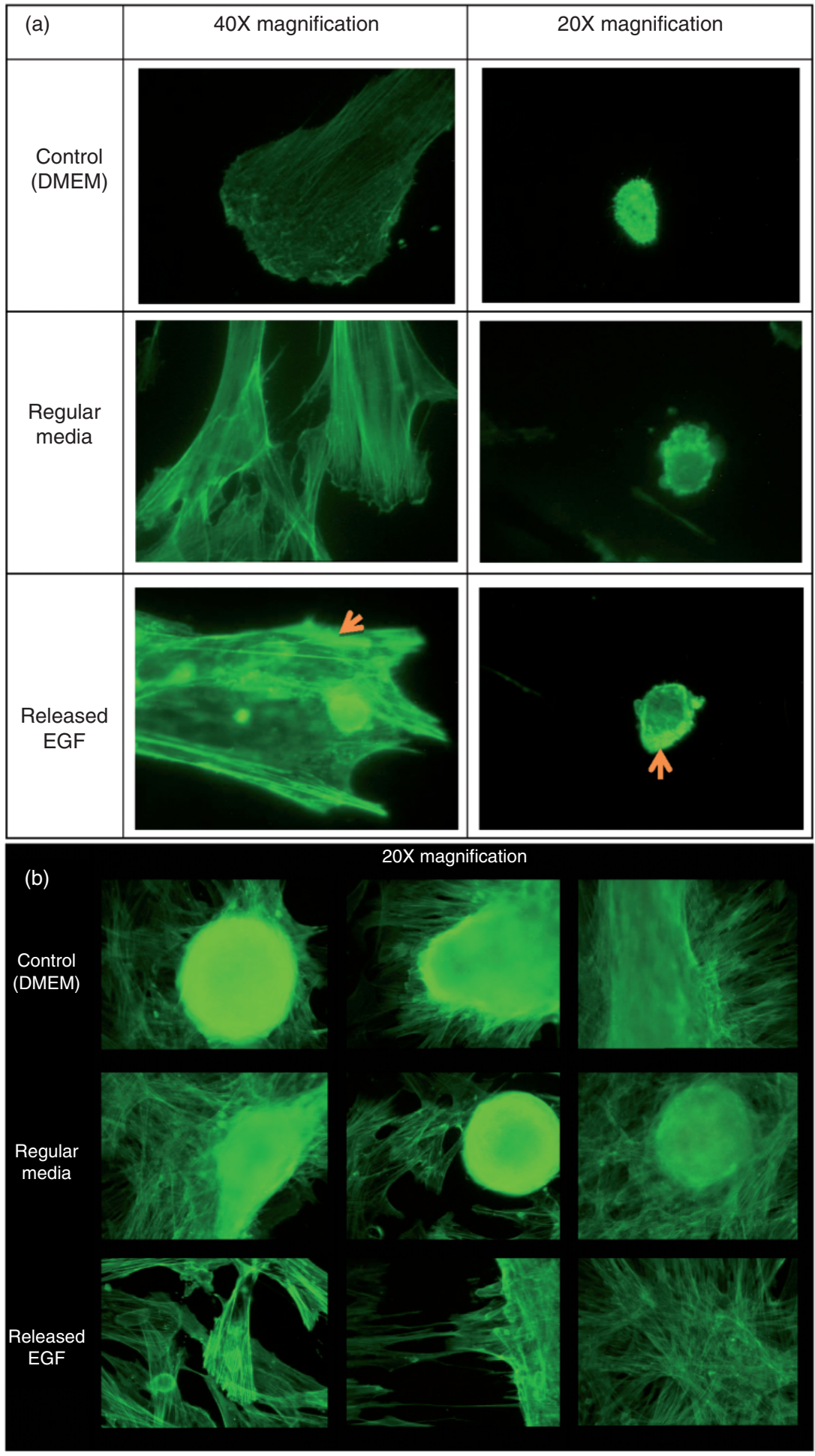

Figure 9. Immunostaining of actin filaments. (a) Differences in F-actin density and distribution. (b) Formation of cellular aggregates. DMEM: Dulbecco's Modified Eagle Medium; EGF: epidermal growth factor.

EGF showed a visible increase in actin filament density compared to the other treatments, which was especially pronounced in the leading edge of polarized cells and in areas of membrane ruffling (Figure 9(a)). Additionally,
F-actin staining highlighted cellular "aggregates" or clumps of fibroblasts (Figure 9(b)) which were more prominent in serum-free and medium-exposed cells, but were markedly reduced in cells incubated in 
released EGF. The differences in F-actin distribution in our study are therefore consistent with both the increased migratory activity we observed, as well as the aggregation of cells in conditions which did not promote cell migration.

\section{Conclusion}

EGF was encapsulated using the LbL assembly on a functionalized surface by pairing up with $\mathrm{CH}$ and subsequently released with a high level of bioactivity. Furthermore, EGF release could be "tuned" for an extended period, depending upon the number of layers deposited onto the surface. Thus, the amount of bioactive EGF released onto a wound site can be adapted on wounds of different depths (i.e., partial thickness or full thickness wounds). Since the LbL method allowed us to well preserve the bioactivity of encapsulated EGF $(>90 \%)$ for its later effective delivery, we expect that this technique will be fruitful tool for the effective fabrication of bioactive wound dressing for expedited wound healing. Taken together, the LbL technique for EGF encapsulation and controlled delivery clearly merits further investigation for clinical use. We plan further refinements of this technique in conjunction with in vivo tests.

\section{Funding}

This work was supported by the Manitoba Health Research Council (MHRC) Operating grant, the Collaborative Health Research Projects (CHRP) operating grant (Grant no.: CHRP 413713-2012), and the Natural Sciences and Engineering Research Council of Canada (NSERC) Discovery grant (Grant no.: RGPIN/372048-2009).

\section{Acknowledgements}

The authors also want to acknowledge Zyme Fast Inc. for kindly donating recombinant human EGF for this study.

\section{References}

1. American Diabetes Association. Consensus development conference on diabetic foot wound care. Mass $A d v$ Wound Care 1999; 12: 353-361.

2. Lavery LA, Ashry HR, Houtum WV, et al. Variation in the incidence and proportion of diabetes-related amputations in minorities. Diabetes Care 1996; 19: 48-52.

3. Edwards JV, Yager D, Bopp A, et al. Design, preparation, and activity of cotton gauze for use in chronic wound research. ACS Symp Ser 2011; 792: 76.

4. Fonder MA and Mamelak AJ. Treating the chronic wound: A practical approach to the care of non-healing wounds and wound care dressings. J Am Acad Dermatol 2008; 58: 185.

5. Menke BN and Diegelmann RF. Impaired wound healing. Clin Derm 2007; 25: 19.
6. Margolis DJ, Kantor J and Berlin JA. Healing of diabetic neuropathic foot ulcers receiving standard treatment. A meta-analysis. Diabetes Care 1999; 22: 692.

7. Deðim Z. Use of microparticulate systems to accelerate skin wound healing. J Drug Targ 2008; 16: 437.

8. Barrandon Y and Green H. Cell-migration is essential for sustained growth of keratinocyte colonies - the roles of transforming growth factor-alpha and epidermal growthfactor. Cell 1987; 50: 1131.

9. Buckley A, Davidson JM, Kamerath CD, et al. Sustained-release of epidermal growth-factor accelerates wound repair. Proc Natl Acad Sci U S A 1985; 8: 7340.

10. Steed DL. The role of growth factors in wound healing. Surg Clinics North Am 1997; 77: 575.

11. O'Keefe E, Batting T and Payne R. Epidermal growth factor receptor in human epidermal cells - direct demonstration in cultured cells. J Invest Dermatol 1982; 78: 482.

12. Tsuboi R and Rifkin DB. Recombinant basic fibroblast growth factor stimulates wound healing in healingimpaired db/db mice. J Exp Med 1990; 172: 245.

13. Papanas $\mathrm{N}$ and Maltezos E. Growth factors in the treatment of diabetic foot ulcers: new technologies, any promise? Int J Low Extrem Wounds 2007; 6: 37.

14. Dong X, Xu J, Wang W, et al. Repair effect of diabetic ulcers with recombinant human epidermal growth factor loaded by sustained-release microsphere. Sci China Ser C - Life Sci 2008; 51: 1039.

15. Niall M, Ryan GB and O'Brien McC. BM. The effect of epidermal growth factor on wound healing in mice. $J$ Surg Res 1982; 33: 164.

16. Buckley A, Davidson JM, Kamerath CD, et al. Epidermal growth-factor increases granulation-tissue formation dose dependently. J Surg Res 1987; 43: 322.

17. Buckley A, Davidson JM, Kamerath CD, et al. Sustained release of epidermal growth factor accelerates wound repair. Proc Natl Acad Sci U S A 1985; 82: 7340-7344.

18. Sheridan RL, Petras L, Lydon M, et al. Once-daily wound cleansing and dressing change: efficiency and cost. J Burn Care Rehabil 1997; 18: 139.

19. Choi JS, Choi SH and Yoo HS. Coaxial electrospun nanofibers for treatment of diabetic ulcers with binary release of multiple growth factors. J Mater Chem 2011; 21: 5258 .

20. Schneider A, Wang XY, Kaplan DL, et al. Biofunctionalized electrospun silk mats as a topical bioactive dressing for accelerated wound healing. EGF containing gelatin-based wound dressings. Acta Biomater 2009; 5: 2570.

21. Ulubayram K, Cakar AN, Korkusuz P, et al. EGF containing gelatin-based wound dressings. Biomaterials 2001; 22: 1345 .

22. Goraltchouk A, Scanga V, Morshead CM, et al. Incorporation of protein-eluting microspheres into biodegradable nerve guidance channels for controlled release. $J$ Cont Rel 2006; 110: 400 .

23. Thula TT, Schultz G, Tran-Son-Tay R, et al. Effects of EGF and bFGF on irradiated parotid glands. Ann Biomed Eng 2005; 33: 685.

24. Macdonald ML, Rodriguez NM, Shah NJ, et al. Characterization of tunable FGF-2 releasing polyelectrolyte multilayers. Biomacromolecules 2010; 11: 2053. 
25. Almodovar J, Bacon S, Gogolski J, et al. Polysaccharidebased polyelectrolyte multilayer surface coatings can enhance mesenchymal stem cell response to adsorbed growth factors. Biomacromolecules 2010; 11: 2629.

26. Kim HS and Yoo HS. In vitro and in vivo epidermal growth factor gene therapy for diabetic ulcers with electrospun fibrous meshes. Acta Biomater 2013; 9: 7371-7380.

27. Liu S, Zhao N and Rudenja S. Surface interpenetrating networks of poly (ethylene terephthalate) and polyamides for effective biocidal property. Macromol Chem Physic 2010; 211: 286.

28. Ivanov VB, Behnisch $J$, Hollander $A$, et al. Determination of functional groups on polymer surfaces using fluorescence labelling. Surf Inter Ana 1996; 24: 251.

29. Suzuki S, Morimoto N and Ikada Y. Gelatin gel as a carrier of platelet-derived growth factors. $J$ Biomater Appl 2013; 28: 595.

30. Cooke JM, Wang Y, Morshead CM, et al. Controlled epi-cortical delivery of epidermal growth factor for the stimulation of endogenous neural stem cell proliferation in stroke-injured brain. Biomaterials 2011; 32: 5688.

31. Hamann MCJ, Tator $\mathrm{CH}$ and Shoichet SM. Injectable intrathecal delivery system for localized administration of EGF and FGF-2 to the injured rat spinal cord. Exp Neuro 2005; 194: 106.

32. Yang Y, Zhao Y, Chen B, et al. Collagen-binding human epidermal growth factor promotes cellularization of collagen scaffolds. Tissue Eng Part A 2009; 15: 3589.

33. Maddala R, Reddy VN, Epstein DL, et al. Growth factor induced activation of Rho and Rac GTPases and actin cytoskeletal reorganization in human lens epithelial cells. Mol Vis 2003; 9: 329.

34. Kumerz M, Heiss EH, Schachner D, et al. Resveratrol inhibits migration and Rac1 activation in EGF- but not PDGF-activated vascular smooth muscle cells. Mol Nutr Food Res 2011; 8: 1230.

35. Rudenja S, Zhao N and Liu S. Surface interpenetrating networks of polyacrylamide in poly(ethylene terephthalate) as a means of surface modification. Eur Polym $J$ 2010; 46: 2078.

36. Araki A, Nakamura H, Nojima N, et al. Stability of recombinant human epidermal growth factor in various solutions. Chem Pharm Bull 1989; 37: 404.

37. Yang C, Huang Y, Wu P, et al. The evaluation of stability of recombinant human epidermal growth factor in burn-injured pigs. Process Biochem 2005; 40: 1661.

38. Kashimata M, Hiramatsu M and Minami N. Biochemical properties of epidermal growth factor in the mouse kidney. Comp Biochem 1987; 86: 651.
39. Magun EB, Planck RS, Matrisian ML, et al. Binding, internalization and intercellular processing of 125Iepidermal growth factor purified by isoelectric focusing. Biochem Biophy Res Comm 1982; 108: 299.

40. Li XF, Feng XQ, Yang S, et al. Effects of molecular weight and concentration of chitosan on antifungal activity against Aspergillus niger. Iranian Polym J 2008; 17: 843.

41. Popat A, Liu J, Lu GQ, et al. A pH-responsive drug delivery system based on chitosan coated mesoporous silica nanoparticles. J Mater Chem 2012; 22: 11173.

42. Quemeneur F, Rinaudo M and Pepin-Donat B. Influence of molecular weight and $\mathrm{pH}$ on adsorption of $\mathrm{CH}$ at the surface of large and giant vesicles. Biomacromolecules 2008; 9: 396.

43. Lundin M, Solaqa F, Thormann E, et al. Layer by layer assemblies of CHand heparin: effect of ionic strength and pH. Langmuir 2011; 27: 7537.

44. Haberska K and Ruzgas T. Polymer multilayer film formation studied by in situ ellipsometry and electrochemistry. Bioelectrochemistry 2009; 76: 153.

45. Kayushina R, Lvov Y, Stepina N, et al. Construction and $\mathrm{X}$-ray reflectivity study of self-assemble. Thin Solid Films 1996; 284-285: 246.

46. Thorne GR, Hrabetova S and Nicholson C. Diffusion of epidermal growth factor in rat brain extracellular space measured by integrative optical imaging. $J$ Neurophysiol 2004; 92: 3471.

47. Ai H, Jones SA and Lvov YM. Biomedical applications of electrostatic layer-by-layer nano-assembly of polymers, enzymes, and nanoparticles. Cell Biochem Biophys 2003; 39: 23.

48. Dogan KA, Gumusderelioglu $M$ and Aksoz E. Controlled release of EGF and bFGF from Dextran hydrogels in vitro and in vivo. $J$ Biomedi Mater Res Part B 2005; 74B: 504.

49. Maeng JH, So JW, Kim J, et al. rhEGF-containing thermosensitive and mucoadhesive polymeric sol-gel for endoscopic treatment of gastric ulcer and bleeding. $J$ Biomater Appl 2014, in press. DOI: 10.1177/ 0885328213499948.

50. Buckley A, Davidson JM, Kamerath CD, et al. Epidermal growth factor increases granulation tissue formation dose dependently. J Surg Res 1987; 43: 322.

51. Kim SJ, McKinnis SV, Nawrocki A, et al. Stimulation of migration and wound repair of guinea-pig airway epithelial cells in response to epidermal growth factor. $\mathrm{Am} \mathrm{J}$ Respir Cell Mol Bio 1998; 18: 66.

52. Ishihara M, Ono K, Sato M, et al. Acceleration of wound contraction and healing with a photocrosslinkable chitosan hydrogel. Wound Repair Regen 2001; 9: 513. 